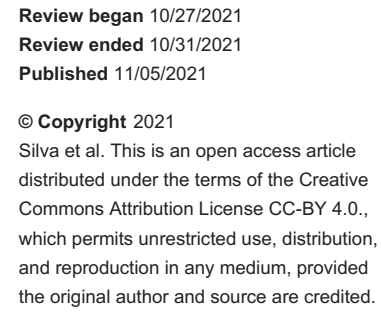

\title{
Role of Imaging in the Management of High-Risk Endometrial Cancer
}

\author{
Catarina Silva $^{1}$, Carolina Carneiro ${ }^{2}$, Teresa Margarida Cunha ${ }^{3}$ \\ 1. Radiology, Hospital Pedro Hispano, Matosinhos, PRT 2. Radiology, Hospital de Braga, Braga, PRT 3. Radiology, \\ Instituto Português de Oncologia de Lisboa Francisco Gentil, Lisboa, PRT
}

Corresponding author: Catarina Silva, catarinaalexandrasilva7@gmail.com

\begin{abstract}
Endometrial cancer (EC) is the second most frequent gynecological malignancy worldwide with an overall favorable prognosis. However, there is a subgroup of patients with a higher rate of recurrence and worse prognosis who benefit from a specific pre- and post-treatment radiological evaluation that allows the adjustment of the therapeutic attitude towards the biology of the tumor.

The main factors that determine high-risk disease are non-endometrioid tumor histology, histopathological grade 3, lymphovascular space invasion (LVSI), myometrial invasion $\geqslant 50 \%$, and cervical stroma involvement. Radiological evaluation helps identify high-risk cases prior to surgical staging and is an important tool both in pre-treatment and in case of clinical recurrence suspicion.
\end{abstract}

As for imaging techniques, both transvaginal ultrasound and MRI can assess local tumor extent while CT and positron emission tomography/CT (PET/CT) help assess lymph nodes and distant metastases.

The central purpose of this article is to review the specific factors that determine high-risk endometrial cancer, and the main specificities in the pre-treatment and follow-up evaluation according to the most recent international guidelines.

Categories: Family/General Practice, Obstetrics/Gynecology, Radiology

Keywords: pet/ct, radiology, mri, gynecological cancer, endometrial cancer

\section{Introduction And Background}

Endometrial cancer (EC) is an increasingly challenging gynecological cancer, being the most common gynecological malignancy in developed countries [1,2]. It is more likely to occur in postmenopausal women, although $20 \%$ to $25 \%$ are diagnosed before menopause [3,3]. Its incidence is increasing worldwide and the risk of EC is positively correlated with obesity and conditions associated with metabolic syndrome [1,4]. In addition, conditions involving excess estrogen, including long-term use of unopposed estrogen, early menarche, and late menopause, predispose women to EC. Tamoxifen, which has pro-estrogenic effects in the uterus, approximately doubles the risk of endometrioid and non-endometrioid types of EC. Parity and oral contraceptive are factors that protect against EC $[2,5,6]$.

Despite its relatively high prevalence, the overall prognosis of EC is favorable with a worldwide low mortality rate estimated at $2.4 \%[2,3,7]$. Approximately $82 \%$ of EC are diagnosed at an early stage of the disease, with abnormal vaginal bleeding or discharge being the initial symptom, which contributes to its global good prognosis [4].

Biologically and clinicopathologically, EC is subdivided into two types $[1,5]$. The type I or estrogendependent carcinomas (>80\%) are composed of grade 1 and 2 endometrioid carcinoma. It is the most common type of EC that may arise from complex atypical hyperplasia and is associated with prolonged unopposed estrogen exposure. The diagnosis of Type I tumors is usually made at earlier stages which confers a relatively good prognosis $[6,8]$.

The type II or estrogen-independent carcinomas account for $10 \%$ to $20 \%$ of EC and are composed of grade 3 endometrioid carcinoma and all non-endometrioid tumors. This type of tumor is less hormone-sensitive and generally more aggressive with a poorer prognosis than type I [6,7-9].

Although EC is surgically staged, it is important to identify the extent of the disease before surgery to optimize the best modality approach and treatment $[7,8,10]$. Preoperative imaging is helpful in patients with suspected extrauterine disease and allows the detection of locoregional advanced disease, identification of suspicious lymph nodes, and distant metastasis [7,11]. Cross-sectional imaging techniques are complementary modalities for surgical evaluation of EC [12] and play an important role both in the pretreatment and follow-up assessment of the disease $[1,7]$. 
The central purpose of this manuscript is to review the role of imaging in high-risk endometrial cancer, particularly in diagnostic and staging assessment, and post-treatment surveillance of these patients.

\section{Review \\ Endometrial cancer stages}

Surgical staging of EC replaced clinical staging by the International Federation of Gynaecology and Obstetrics (FIGO) Committee on Gynecologic Oncology in 1988, and greater knowledge of the tumor biology allowed an update in the FIGO staging system in 2009 [8,12-14]. The complete surgical staging procedure includes hysterectomy with bilateral salpingo-oophorectomy, with biopsies of eventual suspicious peritoneal lesions and cytology of peritoneal washings as well as pelvic and retroperitoneal lymphadenectomy $[8,15]$.

The FIGO stage IA and IB are divided accordingly by the depth of myometrial invasion (less or $\geqslant 50 \%$ myometrial invasion, respectively). Endometrial cancer is classified as stage II if there is cervical stromal invasion, which represents a higher risk of lymphovascular space invasion and is consequently associated with a poorer prognosis. For assigning stage III, local tumor spread must be beyond the uterus but still present within the female pelvis. Subset IIIA represents the invasion of serosa or adnexa involvement while subset IIIB shows direct parametrium or vaginal infiltration. Stage IIIC indicates nodal involvement and is subdivided into pelvic (stage IIIC1) and para-aortic (stage IIIC2) nodal involvement. Stage IV disease represents the direct full-thickness invasion of the bladder or rectal mucosa (stage IVA) or the presence of distant metastases (stage IVB) [6-9,13].

\section{Prognostic tumor characteristics for high-risk disease}

Surgical staging can not be replaced by imaging. However, preoperative risk stratification remains an important tool to predict patient prognosis [4,7]. Furthermore, the selection of preoperative high-risk patients helps define the surgical approach and the need and extent of lymph node sampling or extended lymphadenectomy $[7,10]$. Several studies have demonstrated that recurrence risk after treatment is related to the depth of myometrial invasion, tumor type and tumor differentiation grade, lymphovascular space invasion (LVSI) as well as FIGO stage [16-19]. These prognostic factors allow the definition of recurrent risk groups and guide decision-making worldwide.

Currently, the molecular factors that may influence the prognosis of the disease are under investigation. However, they are still not being used in most cancer centers worldwide [4].

The main criteria to determine high-risk patients are non-endometrioid histology, histopathological grade 3 , LVSI, myometrial invasion $\geqslant 50 \%$, and cervical stroma involvement [6-9]. Tumor histology and grade are determined with endometrial sampling [12]. As for the assessment of myometrial invasion and cervical stroma involvement, MRI can accurately help assess this information [20-22].

High-grade Endometrial Cancer

High-grade EC includes all non-endometrioid tumor histology (mucinous carcinoma, serous carcinoma, clear cell carcinoma, neuroendocrine tumors, mixed cell adenocarcinoma, and undifferentiated or dedifferentiated carcinoma) and histopathological grade 3 (poorly/undifferentiated carcinoma) $[5,6]$.

Lymphovascular Space Invasion

The criterion of LVSI is purely anatomopathological. It may be advanced at the time of biopsy, however, if absent initially, this should be confirmed again at the time of the surgical staging specimen evaluation. Recent data report that LVSI is an independent predictor for local and distant recurrence $[5,8,23]$.

Myometrial Invasion and Cervical Stroma Involvement

Radiology has a central role in determining the presence of these criteria during the pre-treatment evaluation, otherwise unknown until the time of surgery $[24,25]$. The depth of myometrial tumor invasion and the involvement of the cervical stroma are two parameters used for staging EC [25,26] and are important prognostic factors [12,25]. Myometrial invasion is measured from the endo-myometrial junction to the deepest point of invasion $[25,27]$. If the tumor is confined to the corpus uteri, or myometrial invasion is $<50 \%$, the tumor is assigned to stage IA. And if $\geqslant 50 \%$ has been invaded, it is categorized as IB (Figure 1) $[9,28]$. 


\section{Cureus}

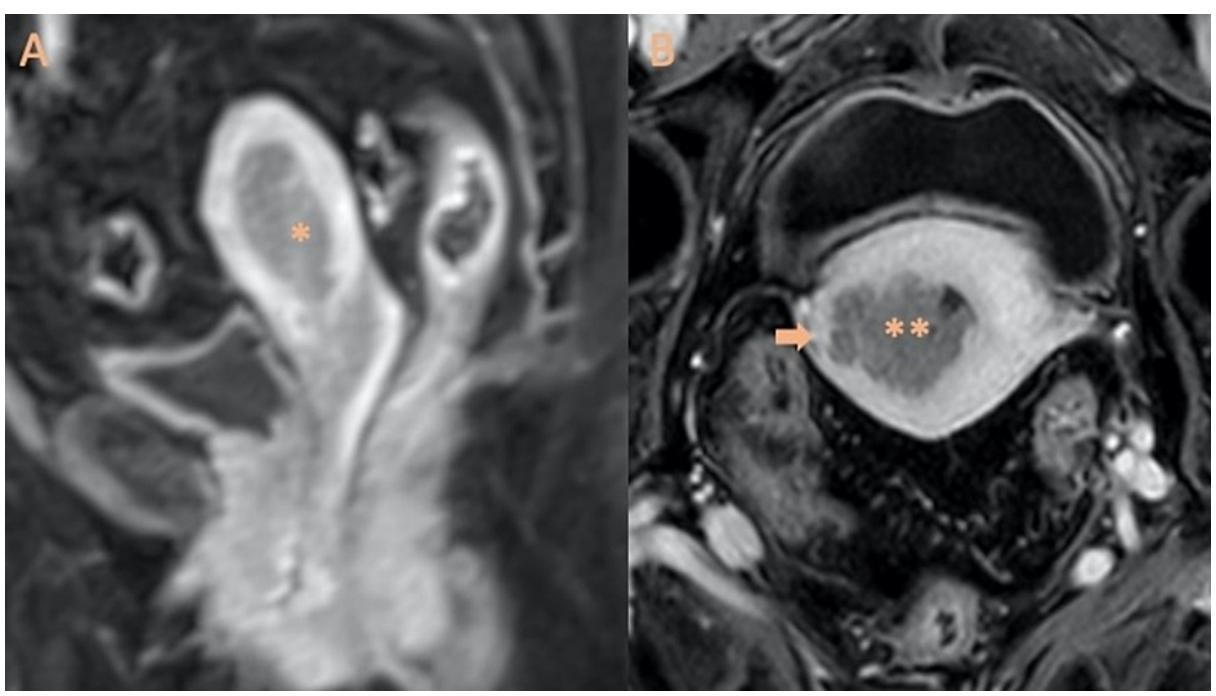

\section{FIGURE 1: Stage I endometrial cancer}

A: Stage IA grade 2 endometrioid adenocarcinoma in a 77-year-old woman. Sagittal T1-weighted MRI with intravenous gadolinium-based contrast material demonstrates endometrial tumor ${ }^{*}$ ) confined to the uterine cavity.

B: Stage IB grade 3 endometrioid adenocarcinoma in an 82-year-old woman. The extent of myometrial invasion is well delineated after administration of intravenous contrast material. The endometrial carcinoma $\left({ }^{* *}\right)$ infiltrates the outer one-half of the myometrium (arrow).

Tumor grade and lymph node metastases correlate with the depth of myometrial invasion, which is the most important morphologic prognostic factor $[11,28]$. Therefore, the degree of myometrial invasion is directly correlated with the probability of advanced disease (in fact, the prevalence of lymph node metastasis is $<2.5 \%$ in stage IA while it ranges from $15 \%$ to $45 \%$ in stage IB) $[7,29,30]$. If the tumor invades the cervical stroma but does not extend beyond the uterus, it is classified as stage II (Figure 2).

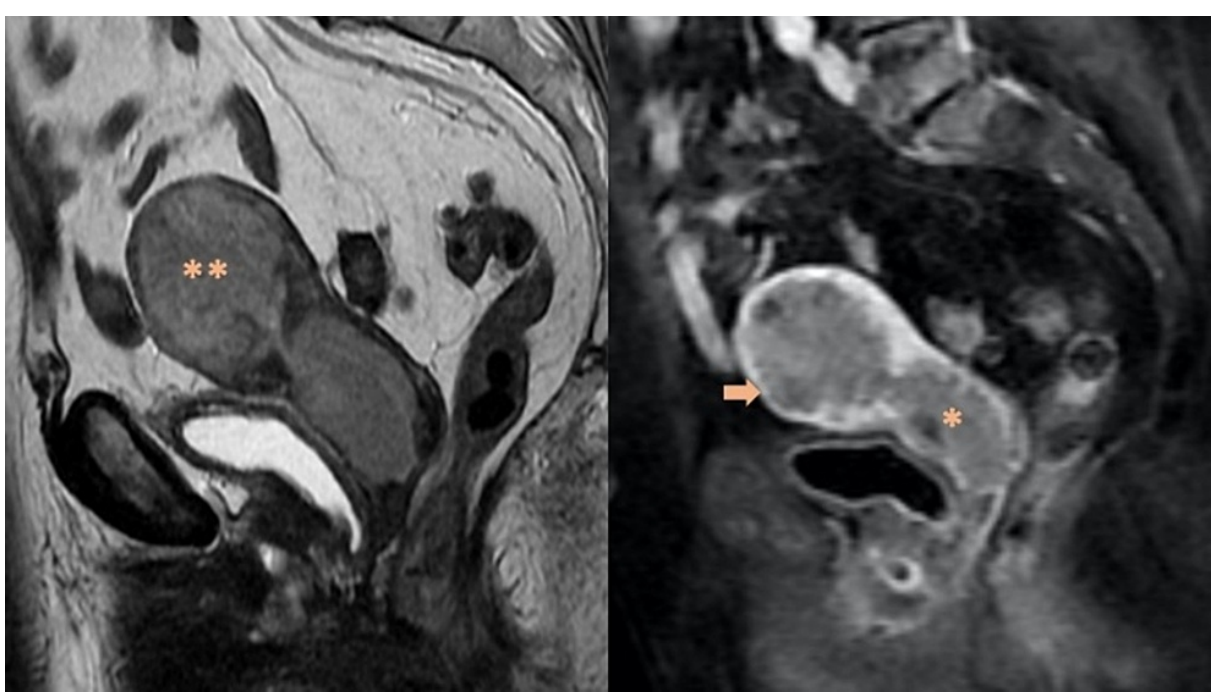

\section{FIGURE 2: Stage II endometrioid adenocarcinoma in an 82-year-} old woman.

Sagittal T2-weighted MRI demonstrates intermediate to high-signal-intensity carcinoma ${ }^{* *}$ ) infiltrating the cervical stroma, which is better delineated after intravenous administration of contrast material $\left.{ }^{*}\right)$. The tumor also invades the outer one-half of the myometrium (arrow).

This stage doesn't include tumors with endocervical glandular involvement, which are classified as stage I, as this has no prognostic impact. The evidence of pretreatment cervical invasion can lead to a different treatment plan and may further lead to preoperative radiation therapy [6]. 


\section{Cureus}

\section{Radiologic evaluation in high-risk patients}

Pre-treatment

Regarding imaging techniques, transvaginal ultrasound remains the first imaging modality for the evaluation of patients with suspected EC. Nevertheless, MRI with intravenous contrast is the primary modality of choice for the anatomical assessment of the pelvic cavity and has an important role in identifying tumor infiltration into myometrium or cervical stroma and extension into parametria [12,25]. With a negative MRI and low-grade tumors, the risk of lymph node metastases is very low. Diffusionweighted MRI (DW-MRI) is useful for detecting small metastatic implants in the omentum or lymph nodes, and also helps in evaluating myometrial infiltration as seen in Figure 3 [28].

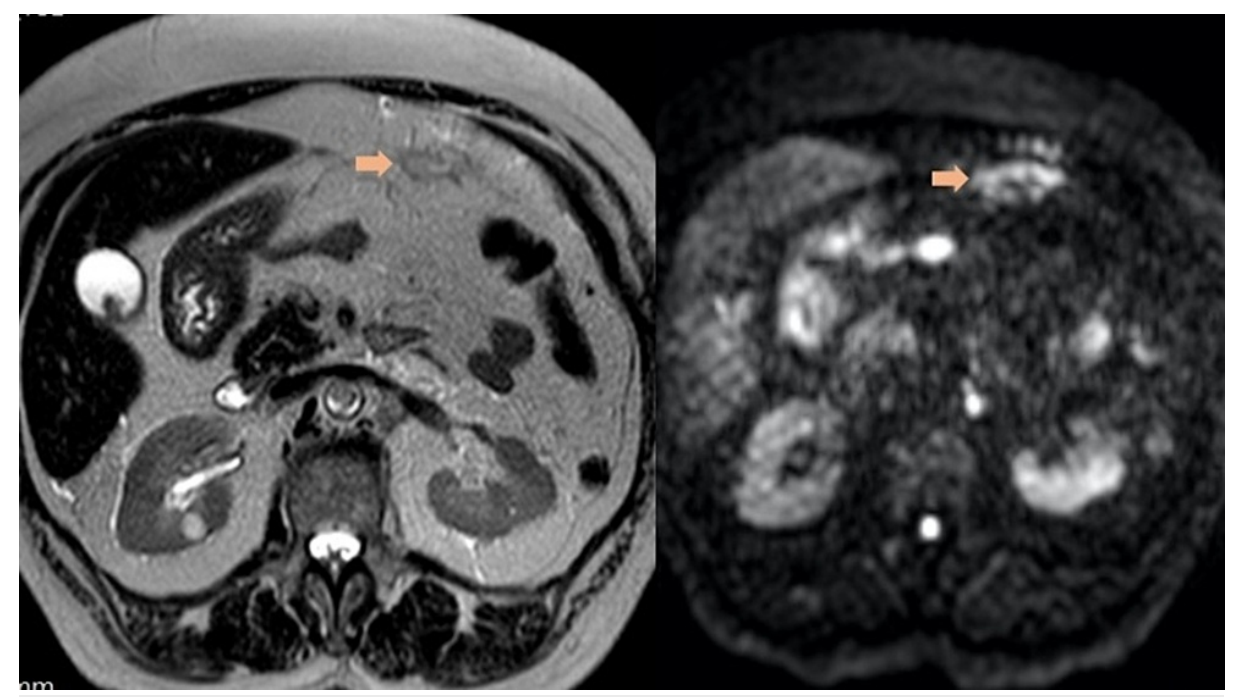

FIGURE 3: Endometrial serous carcinoma in a 67-year-old woman.

Subtle peritoneal deposits (arrow) can be better delineated with diffusion-weighted imaging and appear as an area of restricted diffusion.

Diffusion-weighted sequences are recommended by the European Society of Urogenital Radiology (ESUR) guidelines as part of the MRI protocol of EC diagnosis. The MRI protocol with thin-section high-resolution T2-weighted images, DW, and dynamic contrast-enhanced sequences improves staging patients with EC [13]. Furthermore, these preoperative images help plan surgery and postoperative adjuvant radiotherapy $[12,15-17]$.

As per the National Comprehensive Cancer Network (NCCN) guidelines, MRI should be used in patients with type II EC or, if there's a suspicion of cervical invasion [10-12].

On the other hand, ESUR guidelines also advise MRI in cases of type I EC to distinguish FIGO stage IA from IB since patients in the first case will not require lymphadenectomy. In addition, ESUR also recommends performing MRI in women of childbearing age with grade I EC who wish to preserve their uterus and fertility to help to exclude cervical and myometrial tumor infiltration. In these cases, if EC is confined to the uterine cavity, the possibility of fertility-sparing treatment must be included in the treatment options $[13,28]$. The American College of Radiology (ACR) also recommends MRI as the best modality approach for the initial staging of EC and treatment planning [7].

Besides pelvic MRI to assess locoregional disease, contrast-enhanced CT of the abdomen and pelvis can be used to assess lymph node metastases in high-risk patients with a reported sensitivity and specificity of 30\% to $57 \%$ and $92 \%$ to $98 \%$, respectively $[14,15,30]$. The CT doesn't have much accuracy to characterize an endometrial mass, as it is usually seen as a hypodense lesion or as an abnormally thickened endometrium frequently not distinguished from benign lesions. However, to visualize the entire pelvic and abdominal cavity to search for enlarged lymph nodes or other soft tissue masses, as well as lungs metastases, CT plays a vital role due to its good multiplanar spatial resolution (Figure 4) [12-15]. 


\section{Cureus}

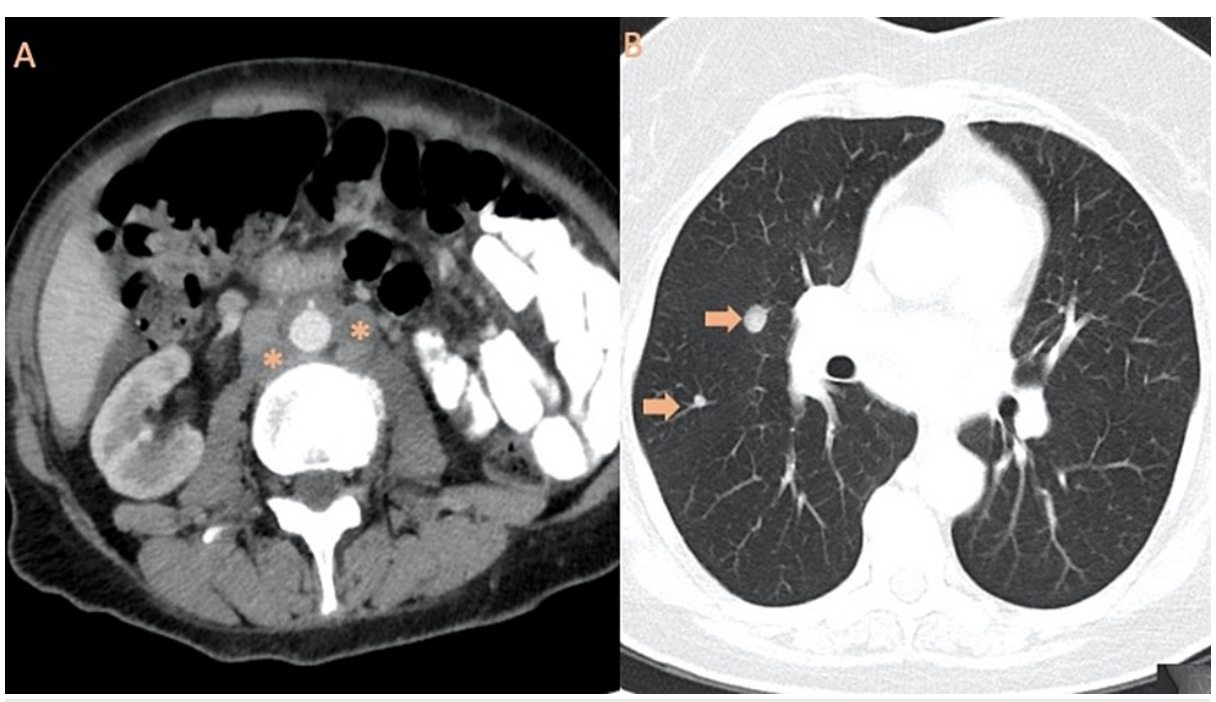

FIGURE 4: CT findings in two different patients with endometrial cancer

A: CT with intravenous contrast in a 54-year-old woman with grade 2 endometrioid adenocarcinoma shows multiple para-aortic lymph nodes metastases $\left(^{*}\right)$ which corresponds to FIGO stage IIIC2.

B: CT of the thorax in a 67-year-old woman with a clear cell carcinoma of the endometrium demonstrates disease distant spread with lung metastases (arrows).

Regarding 18F-fluorodeoxyglucose (FDG) PET/CT, the overall pooled sensitivity and specificity for detection of lymph node metastasis were $72 \%$ and $94 \%$, respectively $[16,17]$. Also, the maximum standardized uptake value (SUVmax) on (FDG) PET/CT is a predictor for overall survival [30-32] and has been correlated with a significantly lower disease-free survival rate. In fact, outcomes of patients with higher FDG uptake were significantly worse than those with lower uptake $[17,18,31]$. Kitajima et al. showed that patients with a high SUVmax $(\geqslant 12.7)$ had a worse prognosis with higher relapse and mortality rates than those with a low SUVmax $(<12.7 ; p=00.00042)$ [19]. The PET/CT has a good correlation with the clinical outcome of the patients and has a high negative predictive value (disease-free courses were reported more frequently in patients with negative PET/CT results) [20,33]. As per the revised ACR guidelines, both PET/CT and contrastenhanced CT of the abdomen and pelvis are adequate imaging techniques for the preoperative evaluation of metastatic disease for a high-grade tumor in the staging of EC [7].

Follow-Up and Recurrent Endometrial Cancer

Approximately one-third of the patients with high-risk cancer may develop recurrent disease and radiology plays a pivotal role in the assessment of locoregional disease, distant metastasis, and the detection of persistence or recurrence of EC $[21,22,30]$. Endometrial cancer tends to recur in the pelvis, especially in the vaginal vault and pelvic lymph nodes, followed by para-aortic lymph nodes. Other common sites for extrapelvic recurrence are the abdomen (especially peritoneum) and lungs [21-23,32]. Regarding surveillance of asymptomatic patients with treated high-risk EC, both radiography chest or chest CT with or without intravenous contrast may be appropriate to search for lung metastases. Besides, contrast-enhanced CT of the abdomen and pelvis can be obtained as a part of posttherapy surveillance in this subgroup of patients [7].

Post-therapy evaluation in patients with a clinically suspected recurrence should include an abdominal and pelvic evaluation. In high-risk patients, it is appropriate to perform PET/CT from the skull base to the midthigh (Figure 5) in addition to the pelvic MRI [7]. 


\section{Cureus}

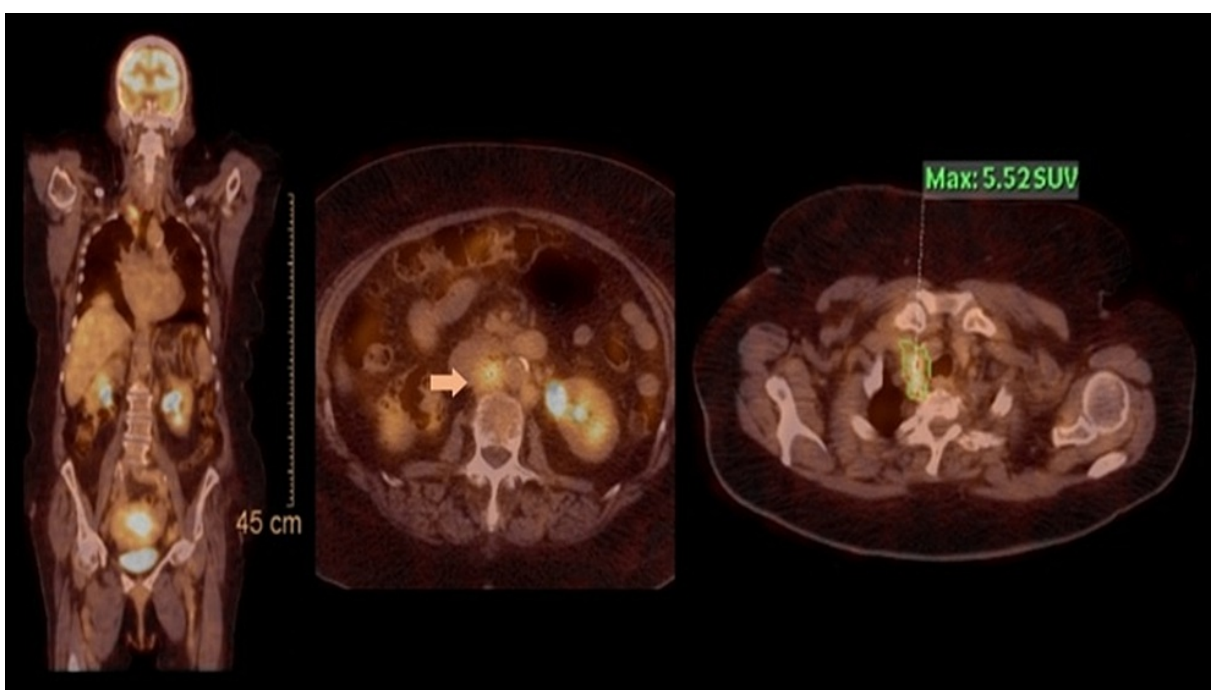

FIGURE 5: PET/CT from skull to mid-thigh performed in the context of clinical suspicion of EC recurrence

Before beginning treatment, an endometrioid carcinoma G2 was diagnosed with the invasion of the outer one-half of the myometrium and vaginal metastasis in a patient. After chemo-radiotherapy, both clinical and radiological responses were obtained. One year post-treatment, the patient performed this PET/CT in the context of recurrence clinical suspicion. A hypermetabolic para-aortic lymph node (arrow) and a right supraclavicular lymph node with a SUVmax of 5.52 (image on extreme right) were detected, conferring the diagnosis of recurrence of the underlying disease.

In a recent meta-analysis, PET/CT has demonstrated a sensitivity and specificity of $95.8 \%$ and $92.5 \%$, respectively, in detecting EC recurrence. Also, literature reports that FDG-PET implemented by CT or MRI has higher accuracy than CT or MRI alone in both evaluations of treatment response and detection of recurrence $[23,32,33]$. The MRI may have a role in the assessment of surgical resectability in the pelvis if it is only local tumoral recurrence. Chest CT with or without intravenous contrast is indicated in high-risk patients to detect lung metastases and is not necessary for low-risk groups as the incidence of lung metastases is very low in these patients [7]. Therefore, to confirm the clinical suspicion of known EC recurrence, CT of the chest, abdomen, and pelvis with intravenous contrast or, PET/CT as well as MRI of the pelvis or abdomen can be used in high-risk patients [7,20].

\section{Conclusions}

Endometrial cancer is a frequent gynecological tumor and although considered to have an overall good prognosis, it is an increasingly significant cause of mortality for women.

High-risk patients are a specific subgroup with a more aggressive clinical course as they are at a higher risk of disseminated disease and recurrence. It includes those with at least one defined radiological or pathological factor, including non-endometrioid histology, histopathological grade 3, lymphovascular space invasion, myometrial invasion $\geqslant 50 \%$, and cervical stroma involvement.

Radiology plays a vital role in the assessment of these patients with MRI frequently used for evaluating locoregional disease, and CT or PET/CT for detecting lymph node involvement and distant metastases. Apart from preoperative staging, imaging is also useful for the detection of postoperative residual disease, detecting recurrent disease as well as in post-treatment surveillance of asymptomatic high-risk patients. These high-risk patients need to be managed by a multidisciplinary team to devise the best treatment approach. Also, radiology can help in patient stratification into treatment groups as well as improve staging accuracy.

In conclusion, radiologists should be aware of the recent advances in the diagnosis and management of endometrial carcinoma, especially in recognizing this specific higher-risk subgroup.

\section{Additional Information}

\section{Disclosures}

Conflicts of interest: In compliance with the ICMJE uniform disclosure form, all authors declare the following: Payment/services info: All authors have declared that no financial support was received from any organization for the submitted work. Financial relationships: All authors have declared that they have 
no financial relationships at present or within the previous three years with any organizations that might have an interest in the submitted work. Other relationships: All authors have declared that there are no other relationships or activities that could appear to have influenced the submitted work.

\section{References}

1. MRI and CT of the Female Pelvis. Forstner R, Cunha TM, Cunha, Hamm B (ed): Springer, Cham, 2019. 10.1007/978-3-319-42575-7

2. Lu KH, Broaddus RR: Endometrial Cancer. N Engl J Med. 2020, 383:2053-2064. 10.1056/NEJMra1514010

3. Sung H, Ferlay J, Siegel RL, Laversanne M, Soerjomataram I, Jemal A, Bray F: Global cancer statistics 2020: GLOBOCAN estimates of incidence and mortality worldwide for 36 cancers in 185 countries. CA Cancer J Clin. 2021, 71:209-249. 10.3322/caac.21660

4. Concin N, Matias-Guiu X, Vergote I, et al.: ESGO/ESTRO/ESP guidelines for the management of patients with endometrial carcinoma. Int J Gynecol Cancer. 2021, 31:12-39. 10.1136/ijgc-2020-002230

5. WHO Classification of Tumours of Female Reproductive Organs . Kurman RJ, Carcangiu ML, Herrington CS, Young RH (ed): IARC Publications, 2014.

6. Amant F, Mirza MR, Koskas M, Creutzberg CL: Cancer of the corpus uteri. Int J Gynaecol Obstet. 2018, 143:37-50. 10.1002/ijgo.12612

7. Reinhold C, Ueno Y, Akin EA, et al.: ACR appropriateness criteria ${ }^{\circledR}$ Pretreatment evaluation and follow-up of endometrial cancer. J Am Coll Radiol. 2020, 17:S472-S486. 10.1016/j.jacr.2020.09.001

8. 53. Corpus uteri - carcinoma and carcinosarcoma . AJCC Cancer Staging Manual, Eighth Edition. Amin MB, Edge SB, Greene FL, et al. (ed): Springer International Publishing, 2017. 661:670.

9. Freeman SJ, Aly AM, Kataoka MY, Addley HC, Reinhold C, Sala E: The revised FIGO staging system for uterine malignancies: implications for MR imaging. Radiographics. 2012, 32:1805-1827. 10.1148/rg.326125519

10. Koh WJ, Abu-Rustum NR, Bean S, et al.: Uterine neoplasms, version 1.2018, NCCN clinical practice guidelines in oncology. J Natl Compr Canc Netw. 2018, 16:170-199. 10.6004/jnccn.2018.0006

11. Beddy P, O'Neill AC, Yamamoto AK, Addley HC, Reinhold C, Sala E: FIGO staging system for endometrial cancer: added benefits of MR imaging. Radiographics. 2012, 32:241-254. 10.1148/rg.321115045

12. Lin MY, Dobrotwir A, McNally O, Abu-Rustum NR, Narayan K: Role of imaging in the routine management of endometrial cancer. Int J Gynaecol Obstet. 2018, 143 Suppl 2:109-117. 10.1002/ijgo.12618

13. Nougaret S, Horta M, Sala E, et al.: Endometrial cancer MRI staging: updated guidelines of the European Society of Urogenital Radiology. Eur Radiol. 2019, 29:792-805. 10.1007/s00330-018-5515-y

14. Connor JP, Andrews JI, Anderson B, et al.: Computed tomography in endometrial carcinoma. Obstet Gynecol. 2000, 95:692-696. 10.1016/s0029-7844(99)00626-2

15. Kitajima K, Suzuki K, Senda M, et al.: Preoperative nodal staging of uterine cancer: is contrast-enhanced PET/CT more accurate than non-enhanced PET/CT or enhanced CT alone?. Ann Nucl Med. 2011, 25:511-519. 10.1007/s12149-011-0496-9

16. Bollineni VR, Ytre-Hauge S, Bollineni-Balabay O, Salvesen HB, Haldorsen IS: High diagnostic value of 18FFDG PET/CT in endometrial cancer: systematic review and meta-analysis of the literature. J Nucl Med. 2016, 57:879-885. 10.2967/jnumed.115.170597

17. Tanaka T, Terai Y, Yamamoto K, Yamada T, Ohmichi M: The diagnostic accuracy of fluorodeoxyglucosepositron emission tomography/computed tomography and sentinel node biopsy in the prediction of pelvic lymph node metastasis in patients with endometrial cancer: a retrospective observational study. Medicine (Baltimore). 2018, 97:e12522. 10.1097/MD.0000000000012522

18. Kitajima K, Kita M, Suzuki K, Senda M, Nakamoto Y, Sugimura K: Prognostic significance of SUVmax (maximum standardized uptake value) measured by $\left[{ }^{18} \mathrm{~F}\right]$ FDG PET/CT in endometrial cancer. Eur J Nucl Med Mol Imaging. 2012, 39:840-845. 10.1007/s00259-011-2057-9

19. Saga T, Higashi T, Ishimori T, et al.: Clinical value of FDG-PET in the follow up of post-operative patients with endometrial cancer. Ann Nucl Med. 2003, 17:197-203. 10.1007/BF02990022

20. Colombo N, Preti E, Landoni F, Carinelli S, Colombo A, Marini C, Sessa C: Endometrial cancer: ESMO clinical practice guidelines for diagnosis, treatment and follow-up. Ann Oncol. 2013, 24:VI33-VI38. 10.1093/annonc/mdt353

21. Kurra V, Krajewski KM, Jagannathan J, Giardino A, Berlin S, Ramaiya N: Typical and atypical metastatic sites of recurrent endometrial carcinoma. Cancer Imaging. 2013, 13:113-122. 10.1102/1470-7330.2013.0011

22. Kadkhodayan S, Shahriari S, Treglia G, Yousefi Z, Sadeghi R: Accuracy of 18-F-FDG PET imaging in the follow up of endometrial cancer patients: systematic review and meta-analysis of the literature. Gynecol Oncol. 2013, 128:397-404. 10.1016/j.ygyno.2012.10.022

23. Meissnitzer M, Forstner R: MRI of endometrium cancer - how we do it . Cancer Imaging. 2016, 16:11. 10.1186/s40644-016-0069-1

24. Sala E, Rockall AG, Freeman SJ, Mitchell DG, Reinhold C: The added role of MR imaging in treatment stratification of patients with gynecologic malignancies: what the radiologist needs to know. Radiology. 2013, 266:717-740. 10.1148/radiol.12120315

25. Kinkel K: Pitfalls in staging uterine neoplasm with imaging: a review . Abdom Imaging. 2006, 31:164-173. 10.1007/s00261-005-0383-8

26. Blaustein's pathology of the female genital tract. Kurman RJ (ed): Springer, New York; 2002. 10.1007/978-1 4757-3889-6

27. Bourgioti C, Chatoupis K, Panourgias E, Tzavara C, Sarris K, Rodolakis A, Moulopoulos LA: Endometrial vs. cervical cancer: development and pilot testing of a magnetic resonance imaging (MRI) scoring system for predicting tumor origin of uterine carcinomas of indeterminate histology. Abdom Imaging. 2015, 40:25292540. 10.1007/s00261-015-0399-7

28. Teng F, Zhang YF, Wang YM, et al.: Contrast-enhanced MRI in preoperative assessment of myometrial and cervical invasion, and lymph node metastasis: diagnostic value and error analysis in endometrial carcinoma. Acta Obstet Gynecol Scand. 2015, 94:266-273. 10.1111/aogs. 12570 


\section{Cureus}

29. Picchio M, Mangili G, Samanes Gajate AM, et al.: High-grade endometrial cancer: value of [(18)F]FDG PET/CT in preoperative staging. Nucl Med Commun. 2010, 31:506-512. 10.1097/MNM.0b013e328337cb47

30. Sohaib SA, Houghton SL, Meroni R, Rockall AG, Blake P, Reznek RH: Recurrent endometrial cancer: patterns of recurrent disease and assessment of prognosis. Clin Radiol. 2007, 62:28-34. 10.1016/j.crad.2006.06.015

31. Chung HH, Kang WJ, Kim JW, Park NH, Song YS, Chung JK, Kang SB: The clinical impact of [(18)F]FDG PET/CT for the management of recurrent endometrial cancer: correlation with clinical and histological findings. Eur J Nucl Med Mol Imaging. 2008, 35:1081-1088. 10.1007/s00259-007-0687-8

32. Nakamura K, Kodama J, Okumura Y, Hongo A, Kanazawa S, Hiramatsu Y: The SUVmax of 18F-FDG PET correlates with histological grade in endometrial cancer. Int J Gynecol Cancer. 2010, 20:110-115. 10.1111/IGC.0b013e3181c3a288

33. Kim HJ, Choi J, Jeong YH, et al.: Prognostic value of metabolic activity measured by (18)F-FDG PET/CT in patients with advanced endometrial cancer. Nucl Med Mol Imaging. 2013, 47:257-262. 10.1007/s13139-0130228-2 